Rhode Island College

Digital Commons @ RIC

Master's Theses, Dissertations, Graduate

Master's Theses, Dissertations, Graduate Research and Major Papers Overview

Research and Major Papers

$12-4-2018$

\title{
Smartphone Apps in Anesthesia: The Next Generation
}

Joshua Tello

Follow this and additional works at: https://digitalcommons.ric.edu/etd

Part of the Nursing Commons

\section{Recommended Citation}

Tello, Joshua, "Smartphone Apps in Anesthesia: The Next Generation" (2018). Master's Theses, Dissertations, Graduate Research and Major Papers Overview. 279.

https://digitalcommons.ric.edu/etd/279

This Major Paper is brought to you for free and open access by the Master's Theses, Dissertations, Graduate Research and Major Papers at Digital Commons @ RIC. It has been accepted for inclusion in Master's Theses, Dissertations, Graduate Research and Major Papers Overview by an authorized administrator of Digital Commons @ RIC. For more information, please contact digitalcommons@ric.edu. 


\title{
SMARTPHONE APPS IN ANESTHESIA: THE NEXT GENERATION
}

by

\author{
Joshua Tello
}

A Major Paper Submitted in Partial Fulfillment

of the Requirements for the Degree of

Master of Science in Nursing

in

The School of Nursing

Rhode Island College

2018 


\begin{abstract}
The number of smartphones in use around the world is increasing daily, as are the apps that are available. Apps are being designed to aid in many aspects of health care practice, including anesthesia, to augment care of patients. The purpose of this study was to ascertain whether student registered nurse anesthetist (SRNAs) enrolled in Rhode Island College School of Nursing/ St. Joseph Hospital School of Nurse Anesthesia program were using smartphones routinely in their practice, if they were using anesthesia-specific smartphone applications (apps), and if so, what types of apps they were using. Rogers’ Diffusion of Innovations (2003) was the theoretical framework that guided this nonexperimental quantitative descriptive study. Following review by the Rhode Island College IRB, 12 participants completed a survey developed by the researcher. Of the respondents, $100 \%$ reported using apps to assist in clinical practice, seven participants reported using drug specific apps more than seven times a week, and nine participants reported using apps for case specific information more than seven times a week. The decision to include anesthesia apps as part of a SRNAs clinical practice is one that each clinician must make based on knowledge, experience, and evidence-based care. However, further research is needed to understand the role of smartphone apps in anesthesia care by SRNAs and CRNAs.
\end{abstract}




\section{Table of Contents}

Background/Statement of the Problem............................................

Literature Review.............................................................

Theoretical Framework............................................................

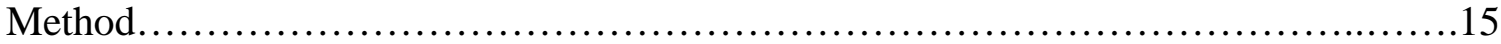

Results........................................................................ 17

Summary and Conclusion.........................................................

Recommendations and Implications for Advanced Nursing Practice...................23

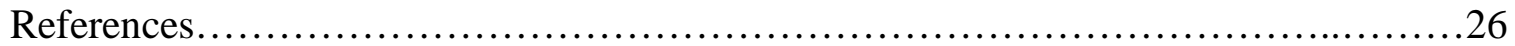

Appendices.................................................................... 30 


\section{Smartphone Apps in Anesthesia: The Next Generation}

\section{Background/Statement of the Problem}

Cellphones are becoming a way of life for people around the globe across all settings. This reliance on cellphones not only affects personal life, but can also have professional implications in the workplace. Cellphones have transformed over the past twenty years to be more than just a means of communication. The majority of cellphone users are now using smartphones, which are capable of much more than what was previously envisioned when thinking of a phone. Smartphones are a way of accessing information, sending messages, viewing images and are used as teaching tools. For the purpose of this paper, the terms smartphone and cellphone will be used interchangeably. The use of Smartphones in the healthcare setting is a topic that has been studied more in recent years because of the number of people who own and use smartphones.

Many healthcare professionals are using smartphones in the workplace to not only access online information for patient care, but also for specialty specific apps to aid in treating patients. In some instances, apps can be used as quick references for drug dosages or treatment algorithms in emergent situations. In the field of anesthesia there has emerged anesthesia specific apps ranging for drug calculations to case tips and references which are being adopted by CRNAs and SRNAs. As smartphone use is becoming more prevalent in healthcare, how this technology is being utilized by anesthesia providers is something that the author of this study hopes to evaluate.

According to Dasari, White, and Pateman (2011), as many as 60\% $(\mathrm{n}=550)$ of anesthesia providers, including physicians and anesthetists, who responded to a survey 
about IPhone use by anesthetists in the professional setting, reported that anesthesiaspecific apps were useful for clinical practice. With technology continuing to develop and the number of smartphones increasing in the U.S., it stands to reason that more healthcare providers own smartphones and may seek to use those smartphones to the fullest of their capabilities while in clinical practice.

The purpose of this study was to ascertain whether student registered nurse anesthetist (SRNAs) enrolled in Rhode Island College School of Nursing/ St. Joseph Hospital School of Nurse Anesthesia program were using smartphones routinely in their practice, if they were using anesthesia-specific smartphone applications (apps), and if so, what types of apps they were using.

Next, the literature review will be presented. 


\section{Literature Review}

A literature review was performed using the databases PubMed, CINAHL, and Medline. Searched keywords included cellphone use in healthcare, cellphone use in nursing, patient care and cellphones, cellphones in the operating room, CRNAs’ cellphone use, smartphones in anesthesia, and anesthesia apps. The time frame searched was from 2007-2017. Fifteen peer-reviewed articles were obtained that were relevant to cellphone use by CRNAs and SRNAs in patient care areas. In these articles, topics included cellphone use by healthcare providers, cellphones as a distraction, perceptions of cellphones in healthcare, anesthesia specific apps, and anesthesia provider smartphone use. For the purpose of this paper, the terms cellphone and smartphone will be used interchangeably.

\section{Cellphone use by Healthcare Providers}

Cellphone use in healthcare has dramatically increased over the past 15 years. Franko and Tirrel (2012) performed a digital survey via email evaluating smartphone and associated app usage amongst members of the Accreditation Council for Graduate Medical Education (ACGME). The results showed that 85\% $(n=317)$ of the respondents used smartphones and 56\% $(n=209)$ reported using specific apps in their clinical practice. Those apps ranged from drug guides, medical calculators, reference material and treatment algorithms to coding and billing apps. The authors concluded that the use of smartphones and apps in the clinical setting will likely continue to increase in the future and more studies should be conducted to assist in the development of future healthcare delivery systems. 
Cellphones are used in the healthcare setting for communication with colleagues as well as accessing information. Koivunen, Niemi, and Hupli (2015) performed a crosssectional survey to describe the means of communication between nurses and other members of the healthcare profession. One hundred twenty-two people responded to the survey and $97 \%(n=118)$ reported commonly using email as communication with colleagues. Text messaging via cellphone was reported as the second most common form of communication between healthcare workers; $61 \%(n=74)$ reported using this method. The nurses in this study reported that using email and text messaging via cellphones not only increased the speed of communication between colleagues, but also increased professional cooperation and made work time more effective. The authors concluded that electronic communication via cellphones would likely increase in the future. They also acknowledged the risk that there may be a decrease in face-to-face contacts, which is an important part of professional growth and knowledge sharing.

The use of cellphones for healthcare is not only seen in western civilizations nor is it limited to healthcare workers alone. According to Steury (2016), who performed a randomized control trial with 96 adult malaria patients in Zambia, cellphones can be used to improve healthcare by both providers and patients. The author used text messages via cellphones to remind patients to take anti-malarial medications and called these reminders an electronic pillbox. The control group participants were given the malaria medication with written instructions on how and when to take the pills. The intervention group received text message reminders to take the dose of medications at the scheduled time. The results showed that 20 participants had an estimated perfect adherence to taking the medication, once the participant returned the number of pills they had left. These 
were then counted and correlated with text messages sent for doses to take; 12 pills (66.7\%) had been taken in the intervention group and eight pills (33.3\%) had been taken in the control group. The intervention group took more of the antimalarial medication than the control group but the author felt that more studies should be conducted in this area.

\section{Cellphones as a Distraction}

Cellphones as a distraction were examined by Hyman, Boss, Wise, McKenzie, and Caggiano (2009). This research study examined pedestrians who talked on cellphones in two scenarios. The researchers followed a total of 347 pedestrians: some listening to music; some talking to friends; some on cellphones; and some without distractions. In the first scenario, it was found that pedestrians on cellphones changed directions more often, walked slower, and were frequently zigzagging off course. In the second scenario, the researchers placed a clown in red polka dots on a unicycle in the middle of the square. After the pedestrians traversed the square they were questioned by the researchers whether or not they saw anything unusual. Of the pedestrians listening to music, 33.3\% $(n=24)$ reported seeing a clown on a unicycle. Of those walking with friends, $58 \%(n=49)$ reported seeing the clown. However, of the pedestrians on a cellphone only $8 \%(n=7)$ spontaneously remembered seeing the clown on the unicycle. The authors concluded that even while performing tasks as simple as walking, a person has a huge loss of awareness of the environment around them while on a cellphone.

Smith, Darling, and Searles (2011) conducted a survey of perfusionists using cellphones during cardiopulmonary bypass. The researchers posted a 19 question survey 
on Perflist and Perfusion.com forums; they received 439 responses including chief perfusionists and staff perfusionists ranging from ages 20 to 60. Of those responses, $55.6 \%(n=244)$ of perfusionists reported using cellphones during the performance of cardiopulmonary bypass; 7.3\% $(\mathrm{n}=32)$ admitted that personal distraction by a cellphone negatively impacted their performance. The number of those who reported witnessing another perfusionists distracted with a cellphone during cardiopulmonary bypass was 33.7\% $(\mathrm{n}=148)$. Of those who responded, $78.3 \%(\mathrm{n}=342)$ reported feeling that cellphones can present a potentially significant safety risk to patients. The authors concluded that though a large number of perfusionists surveyed believe distractions from cellphones raise safety issues, the majority of respondents admitted to using a cellphone during cardiopulmonary bypass.

Cellphones as a distraction have been seen in many settings, most notably in highway safety. According to the National Safety Council (2015), 20\% of all motor vehicle crashes (1.1 million crashes) in 2013 in the United States involved cellphones.

\section{Perceptions of Cellphone Use by Healthcare Providers}

Only two studies were found on the perceptions of cellphone use by healthcare providers thus there is a wide gap between the years of the following studies. Garrett and Klein (2008) examined the value of wireless personal digital assistants in practice and perceptions of advanced practice nurses (APNs). The aim of their study was to evaluate APNs perception of wireless personal assistant technologies, establish the type of wireless tools, and determine how they are useful in practice. An inductive qualitative approach was employed to survey APNs via convenience sampling using questionnaires 
and focus group interviews. A total of 73 questionnaires were mailed and 43 were returned. Twenty-four nurse practitioners (NPs) were interviewed. Results from the study showed that APNs felt that personal digital devices improved access to specialist knowledge, protocols, and even consulting for patient-centered decision making. They also voiced that having the ability to access drug references, prescribing medications from their devices, and having the ability to wirelessly input data was a major factor in their practice and significantly improved client care.

McNally, Frey, and Crossan (2016) performed a qualitative descriptive study aimed at understanding the perceptions of the application of personal hand-held referencing at the bedside. The study took place in New Zealand and explored perceptions of nurse managers and student nurses. To obtain the data, purposeful sampling was used that included five nurse managers and 13 student nurses, representing $6.5 \%$ of the 200 total students. The researchers conducted two semi-structured interviews, one at New Zealand Regional Polytechnic Nursing School and the other at the main district hospital. While student nurses $(n=13)$ thought that smartphones and app technology offered countless resources to aid in their educational experience, they thought that there was a perception of unprofessionalism when used in clinical settings. Nurse managers $(\mathrm{n}=5)$ theoretically supported the idea of handheld referencing technology and its use to improve patient care. However, there was much concern surrounding the unregulated nature of personal smartphones and the perceived lack of professionalism. The consensus of the managers was that they did not approve the use of smartphones at the bedside. 


\section{Anesthesia Provider Smartphone Use}

Dasari et al. (2011) conducted a survey of IPhone use among anesthetists in England asking about medical apps and anesthesia apps for educational purposes. Of the 918 responses received, 80\% $(n=734)$ used their IPhone for medical apps and 60\% $(n=$ 551) reported using anesthesia specific apps. Using an app for educational purposes represented 47\% $(n=431)$ of usage. However, of the anesthetist surveyed, only $8 \%(n=$ 73) reported being technologically savvy anesthetists. The researchers acknowledged that the number of providers using smartphones in practice will likely continue to increase and encouraged future app development by anesthetists to improve patient safety, medical education, and overall patient experience.

A questionnaire consisting of 14 questions was used by Pinar, Karaca, Dogan, and Konuk (2016) to survey anesthesia practitioners in Turkey regarding the use of smartphones during anesthesia care. The questionnaires were sent out via email and handed out in a hard copy form at a national meeting for anesthetists. The response rate ranged from $19 \%$ of hard copy questionnaires (355 responses) to $22 \%$ of emailed (600 responses). Of those responses, 251 (26.2\%) were attending anesthesia physicians, 311 (32.5\%) were medical residents in anesthesia, and 325 (34 \%) were nurse anesthetists. The results of the study showed that $93.7 \%(n=894)$ of those who responded admitted to using smartphones during anesthetized patient care. The frequency of smartphone use during patient care significantly decreased in providers over the age of 40 as compared to providers aged 20 to 40 . There was also a vast difference in the amount of smartphone use depending on the stage of anesthesia. There was a dramatic decrease in use during critical stages; $77 \%(n=741)$ of those questioned reported never using smartphones 
during anesthesia induction and emergence. The most common reason cited for cell phone use was for communication, with $65.4 \%(n=625)$ of usage for phone calls and 46.4\% ( $\mathrm{n}=443)$ for messaging. These included personal and in-hospital communication. The researchers acknowledged that in the age of evidence-based practice, banning smartphone use while providing anesthesia care would not be appropriate, however distractions from cellphones drastically strain anesthesia personnel.

\section{Anesthesia-Specific Apps}

Low et al. (2011) conducted a randomized control trial to determine the benefit of using iResus, an advanced life support application that simulated medical emergencies. The authors evaluated 31 participants, ranging from general practitioners to anesthesiologists, who had been trained in advanced life support over the past 48 months. The participants were randomly split into a control group with no smartphone use and a test group using the iResus smartphone app. Both groups were tested with a cardiac arrest simulation test that used a validated scoring system. The test was scored on a grading scale 0 to 100 and this score was the outcome measured for the study. The results showed that the median score for the control group was 72 and 84.5 for the test group. The results indicated that using the iResus application improved provider's confidence and preparedness for medical emergencies.

In an era in which cellphones are such an integral part of society, many anesthesia providers are using cellphone apps to complement their practice. One example of this type of app usage is seen in the randomized clinical trial performed by Liguori et al. (2016). The goal of the study was to measure preoperative anxiety. The authors aimed to 
evaluate the effectiveness of an app in reducing preoperative anxiety in children. It was a non-blinded, randomized control trial which included 40 children ages 6 to 11 years-old who were about to undergo a non-emergent surgical procedure. The intervention used was an app that could be accessed on a smartphone or tablet. The app was video showing two physicians dressed as clowns touring the operating room and explaining what to expect in a joking manner. The possible range of anxiety scores ranged from a minimum of 23.33 to a maximum anxiety score of 100 . The initial mean anxiety score for the control group was 37.1 and 37.3 for the experimental group. Upon entering the operating room, the anxiety of the subjects was tested again. The controlled group scored 48.6 and the experimental group 33.0. The conclusion was that use of the app reduced preoperative anxiety in children admitted to the National Health System Pediatric Hospital.

In another study, Gupta and McEvoy (2016) created an app in collaboration with Vanderbilt University and the American Society of Regional Anesthesia and Pain Medicine (ASRA) to be a support tool for decision-making based on the 2010 published guidelines for patients receiving regional anesthesia while on anticoagulation. The app provided an algorithm for administering regional anesthesia to patients on anticoagulation therapy. The number of users who downloaded the app and searched data was collected worldwide between April 2014 and October 2015, with their consent. The results showed that 8,381 downloads were made, and of those, $81 \%(n=6 ; 789)$ were in the United States. Approximately 30,000 individual searches were performed from 4,503 different devices. The most commonly searched medication was rivaroxaban $(n=4,427 ; 11 \%)$ and neuraxial procedures were the most commonly searched procedures $(n=22,477 ; 78 \%)$. The researchers concluded that there is a need for future app updates for use in clinical 
anesthesia practice and that smartphone apps are a valuable mechanism for distributing anesthesia guidelines and updates rapidly.

Next, the theoretical framework will be presented. 


\section{Theoretical Framework}

Rogers’ Diffusion of Innovations (2003) is the theoretical framework that will guide this non-experimental quantitative descriptive study. "Diffusion is the process by which an innovation is communicated through certain channels over time among the members of a social system” (Rogers, 2003, p. 5). Rogers also postulated that each member of a society must make his/her own decision because decisions are not made collectively or authoritatively.

According to Rogers (2003), in making those decisions, each individual will go through a five step process. Step 1 is knowledge; the decision maker is exposed to the innovation and gains knowledge of its function. During step 2, the process is known as persuasion; the decision maker formulates a favorable or unfavorable approach towards the innovation. Step 3 is the decision stage in which the decision maker approves or rejects the innovation. Implementation is step 4, where the decision maker begins to use the innovation. Finally, step 5 is confirmation; the decision maker seeks support of the innovation already implemented or reverses the decision to approve or reject the innovation.

Rogers (2003) further postulated that the decision to implement innovations is based on others in the social system and a cost benefit ratio. The person must determine if the benefits of adopting a new innovation outweigh the risk of said innovation. In making that decision, the person's individual diversity and characteristics are what lead to the diffusion of the innovation. Because a person's temperament and background play a role 
in this process, the innovativeness of the person making the decision to implement the innovation must be assessed as well.

According to Rogers (2003), innovativeness can be defined as the rate at which an individual adopts a new idea earlier than other members of a system. This innovativeness can be classified into five categories. Innovators are the first ones to adopt a new innovation and want to be cutting edge. Early adopters, who use information provided by innovators to form their decision on whether or not to implement the innovation, are considered to make well informed decisions about new innovations. Early majority people will consider adopting an innovation after a longer amount of time. Late majority individuals will adopt the innovation after the average person in the group has adopted it, not wanting to be the first to try a new innovation. Laggards are the last group and are considered to be traditionalist or stand-alone individuals in the social system, they are weary of change and thus are suspicious of new innovations.

Putzer and Park (2010) used the Diffusion of Innovation as part of a framework for a study on the effects of innovation factors on smartphone adoption by nurses in community hospitals. They surveyed 200 nurses at two different community hospitals with a $38 \%(n=76)$ response rate. In their study, they viewed smartphones as a recent innovation and used Roger's theory to hypothesize seven relationships between nurse's attitudes towards using smartphones and factors that may influence smartphone use. The authors concluded that the decision to adopt smartphones as an innovation in nursing was affected based on having characteristics of observability, job relevance, compatibility, and internal and external influences that affect attitudes about using smartphones. 
Applying Rogers’ Diffusion of Innovation (2003), the author of this study will frame smartphones and specifically applications on smartphones as an innovation that SRNAs are using in their practice. Using a survey, the author will examine how many are using this innovation currently, what types of applications they are using and how often they are being used in practice.

Next, the study methods will be presented. 


\section{Method}

\section{Purpose}

The purpose of this study was to ascertain whether student registered nurse anesthetist (SRNAs) enrolled in Rhode Island College School of Nursing/ St. Joseph Hospital School of Nurse Anesthesia program were using smartphones routinely in their practice, if they were using anesthesia-specific smartphone applications (apps), and if so, what types of apps they were using.

\section{Design}

The design of this descriptive study was non-experimental mixed method survey, with qualitative and quantitative data.

\section{Sample/Site}

Convenience sampling was used to recruit a sample of student registered nurse anesthetist SRNAs enrolled in RIC School of Nursing/ St. Joseph Hospital School of Nurse Anesthesia program from 2017 through 2018. Exclusion criteria was students enrolled in nurse anesthesia programs who have not started clinical practice at the time of the survey distribution.

\section{Method}

Upon review by the Rhode Island College IRB, an informational consent letter, a recruitment letter, and link to the survey was emailed to the program director of St. Joseph Hospital School of Anesthesia for Nurses. The director was asked and agreed to forward the informed consent and survey link to the students enrolled in the nurse 
anesthesia program from 2017 to 2018. Potential participants were instructed to review the informational letter and if they wished to participate in the survey, they clicked on an embedded link. Survey Monkey® ${ }^{\circledR}$ was used as a delivery service and the IPE addresses of the participants were not collected. Potential participants were assured that their participation was voluntary and that their responses remained anonymous and confidential.

\section{Measurement}

The survey included seven multiple choice questions and one short answer question. The survey was created by the author of this study. The design of the questions was based on the author's personal experiences with smartphone apps, their use in clinical practice, and the literature. Prior to disseminating the survey, it was piloted with a small group of CRNAs to review the questions' understandability and clarity; minor modifications were made based on the group’s feedback.

\section{Data Analysis}

The data were analyzed using basic descriptive statistics for mean scores and percentages based on how often SRNAs are using smartphone apps in clinical practice and what types of apps were being used.

Next, the results will be presented. 


\section{Results}

The survey was forwarded by the program director of St. Joseph Hospital School of Nurse Anesthesia to 19 possible participants; 12 participants completed the online survey between June 2018 and July 2018, with a response rate of 64\%. Table 1 summarizes the responses to the first five questions.

Of the 12 SRNAs who completed the survey, $100 \%(n=12)$ of them reported using apps on smartphones to assist in clinical practice. One-third $(\mathrm{n}=4)$ reported using apps to assist in clinical practice and $25 \%(\mathrm{n}=3)$ responded that they used apps 20 or

more times a week. The use of apps for drug reference during clinical practice ranged in responses as high as more than 20 times a week $(8.33 \%$; $n=1)$ to as low as zero uses per week $(8.33 \%$; $n=1)$, with $25 \%(n=3)$ reporting uses seven to 10 times a week. When asked about using apps for specific case tips or pointers, 33.33\% ( $n=4)$ reported usage between 10 and 20 times a week. The use of apps for specialty anesthesia such as pediatrics varied between one to 20 times a week; $58.33 \%(n=7)$ responded N/A due to not rotating to pediatrics by the time they completed the survey. The use of apps for regional anesthesia was reported as being used one to three times a week by 33.33\% $(n=2)$ and $16.67 \%$ reported use four to six times a week. 


\begin{tabular}{|c|c|c|c|c|c|c|c|}
\hline Question & 0 & $1-3$ & $4-6$ & $7-10$ & $10-20$ & $\begin{array}{l}20 \text { or } \\
\text { more }\end{array}$ & N/A \\
\hline $\begin{array}{l}\text { How many } \\
\text { times a week do } \\
\text { you use apps on } \\
\text { your } \\
\text { smartphone to } \\
\text { assist in clinical } \\
\text { practice? }\end{array}$ & $0 \%$ & $\begin{array}{l}8.33 \% \\
(n=1)\end{array}$ & $\begin{array}{l}8.33 \% \\
(n=1)\end{array}$ & $\begin{array}{l}33.33 \% \\
(n=4)\end{array}$ & $\begin{array}{l}25 \% \\
(n=3)\end{array}$ & $\begin{array}{l}25 \% \\
(n=3)\end{array}$ & ------ \\
\hline $\begin{array}{l}\text { How many } \\
\text { times in one } \\
\text { week do you } \\
\text { use apps for } \\
\text { drug } \\
\text { references? }\end{array}$ & $\begin{array}{l}8.33 \% \\
(n=1)\end{array}$ & $\begin{array}{l}16.67 \% \\
(n=2)\end{array}$ & $\begin{array}{l}16.67 \% \\
(n=2)\end{array}$ & $\begin{array}{l}25 \% \\
(n=3)\end{array}$ & $\begin{array}{l}25 \% \\
(n=3)\end{array}$ & $\begin{array}{l}8.33 \% \\
(n=1)\end{array}$ & ------ \\
\hline $\begin{array}{l}\text { How many } \\
\text { times per week } \\
\text { do you use apps } \\
\text { for case } \\
\text { information } \\
\text { (specific case } \\
\text { tips or } \\
\text { pointers)? }\end{array}$ & $\begin{array}{l}8.33 \% \\
(n=1)\end{array}$ & $\begin{array}{l}8.33 \% \\
(n=1)\end{array}$ & $\begin{array}{l}8.33 \% \\
(n=1)\end{array}$ & $\begin{array}{l}25 \% \\
(n=3)\end{array}$ & $\begin{array}{l}33.33 \% \\
(n=4)\end{array}$ & $\begin{array}{l}16.67 \% \\
(n=2)\end{array}$ & ------ \\
\hline $\begin{array}{l}\text { How often do } \\
\text { you use } \\
\text { pediatric } \\
\text { specific } \\
\text { anesthesia } \\
\text { apps? If you do } \\
\text { not do pediatric } \\
\text { anesthesia, } \\
\text { please put N/A. }\end{array}$ & $0 \%$ & $\begin{array}{l}8.33 \% \\
(n=1)\end{array}$ & $\begin{array}{l}16.67 \% \\
(n=2)\end{array}$ & $0 \%$ & $\begin{array}{l}16.67 \% \\
(n=2)\end{array}$ & $0 \%$ & $\begin{array}{l}58.33 \% \\
(n=7)\end{array}$ \\
\hline $\begin{array}{l}\text { How many } \\
\text { times a week do } \\
\text { you use apps } \\
\text { for regional } \\
\text { anesthesia? If } \\
\text { you do not } \\
\text { perform } \\
\text { regional } \\
\text { anesthesia, } \\
\text { please indicate } \\
\text { N/A }\end{array}$ & $\begin{array}{l}8.33 \% \\
(n=1)\end{array}$ & $\begin{array}{l}33.33 \% \\
(n=4)\end{array}$ & $\begin{array}{l}16.67 \% \\
(n=2)\end{array}$ & $0 \%$ & $0 \%$ & $0 \%$ & $\begin{array}{l}41.67 \% \\
(n=5)\end{array}$ \\
\hline
\end{tabular}


Responses to survey questions 6 and 7 are illustrated in Table 2 below.

\begin{tabular}{|l|l|l|l|}
\hline Questions & Yes & \multicolumn{2}{|l|}{ No } \\
\hline Have you ever paid for & $91.67 \%,(\mathrm{n}=11)$ & \multicolumn{2}{|l|}{ No: 8.33\% (n=1) } \\
\hline $\begin{array}{l}\text { How many apps } \\
\text { specific to your }\end{array}$ & $1-3$ & $4-6$ & $7-10$ \\
anesthesia practice do & & $25 \%(\mathrm{n}=3)$ & $8.33 \%(\mathrm{n}=1)$ \\
you have on your & & & \\
smartphone? & & & \\
\hline
\end{tabular}

When asked if participants had ever paid for an anesthesia app, 91.67\% (n=11) responded “yes”. Two-thirds $(n=8)$ reported having one to three anesthesia apps on his/her phone at the time of completing the survey. The final question on the survey asked participants to list their most commonly used anesthesia apps; 91.67\% (n=11) reported using Vargo. Other apps listed included Medscape, Lab Value Pro, Anesthesia Drugs Fast, Block Buddy, CRNA Pocket Prep, IBM Drug info, and Up To Date.

Next, summary and conclusion will be presented. 


\section{Summary and Conclusions}

Cellphones are becoming a way of life for people around the globe across all settings. Many healthcare professionals are using smartphones in the workplace to not only access online information for patient care, but also for specialty specific apps to aid in treating patients. Franko and Tirrel (2012) performed a digital survey via email evaluating smartphone and associated app usage amongst members of the Accreditation Council for Graduate Medical Education (ACGME). The results showed that 85\% (n = 317) of the respondents used smartphones and 56\% $(n=209)$ reported using specific apps in their clinical practice. In the field of anesthesia, there have emerged anesthesiaspecific apps ranging from drug calculations to case tips and references which are being adopted by CRNAs and SRNAs. Pinar et al. (2016) surveyed anesthesia practitioners in Turkey regarding the use of smartphones during anesthesia care. The results of the study showed that $93.7 \%(n=894)$ of those who responded admitted to using smartphones during anesthetized patient care. The purpose of this study was to ascertain whether student registered nurse anesthetist (SRNAs) enrolled in Rhode Island College School of Nursing/ St. Joseph Hospital School of Nurse Anesthesia program were using smartphones routinely in their practice, if they were using anesthesia-specific smartphone applications (apps), and if so, what types of apps they were using.

Rogers’ Diffusion of Innovations (2003) was the theoretical framework that guided this non-experimental quantitative descriptive study. "Diffusion is the process by which an innovation is communicated through certain channels over time among the members of a social system” (p. 5). The author of this study framed smartphones and specifically applications on smartphones as an innovation that SRNAs are using in their 
practice. A survey was developed based on the literature and the author's personal experience with smartphone apps and their use in clinical practice.

After IRB review was obtained, a recruitment and an informational letter were sent to the program director of St. Joseph Hospital School of Nurse Anesthesia, who then forwarded the letter to potential participants. A convenience sample of 12 SRNAs agreed and completed the survey, which was anonymously completed via the online platform Survey Monkey. The results demonstrated that $100 \%$ of those surveyed reported using apps on their smartphones to assist in clinical practice. Respondents reported using apps in practice for a variety of reasons and some reported using those apps as much as 20 times a week or more. The types of apps used depended on clinical experience and which specialties had been completed at the point of taking the survey.

A small sample size was one of the limitations of this study. There was also no specific information found in the literature about anesthesia specific-apps being used by SRNAs. The author of the survey constructed questions on areas presented in the literature. The survey was pilot tested on a small group of SRNAs for clarity but no further efforts were made to validate the survey. The study also did not differentiate between those just beginning their clinical practice and those who have been in clinical practice for more than a year.

In conclusion, the results identified several interesting points about usage of anesthesia apps by SRNAs. All of the participants found it important to use smartphone apps for clinical practice, some multiple times a day, with the vast majority being willing to pay for those apps costing as much as approximately $\$ 80$. It was clear that the SRNAs 
enrolled in Rhode Island College School of Nursing/St. Joseph Hospital School of Nurse Anesthesia program were willing to incorporate anesthesia apps into their clinical practice and believed them to be important enough to use multiple times a day.

Next, recommendations and implications for advanced practice will be presented. 


\section{Recommendations and Implications for Advanced Nursing Practice}

Advanced Practice Nurses (APNs) play an important role in the landscape of healthcare. As technology progresses, it brings with it changes to that landscape that affect APNs. Further research should be done to evaluate how often other practitioners are using apps in clinical practice, the types of apps, and if there is a common app that is being used routinely in practice amongst different disciplines. Advanced practice nurses are incorporating new forms of technology into their practice and smartphone apps are becoming not only acceptable but alse expected of many APNs. Rogers’ Diffusion of Innovations (2003) postulated that each member of a society must make his/her own decision because decisions are not made collectively or authoritatively. Accordingly, the method that SRNAs and CRNAs use to obtain information differs by provider. With the advancement in smartphone capabilities and the number of anesthesia apps increasing, the results of this research illustrated how the decision to use this innovation in practice is impacting SRNAs in one program.

Adoption of new innovations into practice can be challenging for nurses at any level. It is important for CRNAs to ensure that evidence-based practice guides their decision making and overall practice. Evidence-based practice should guide multiple team members from different specialties, including not only the latest science but also technology. It is this model that encourages SRNAs and CRNAs to incorporate new innovations into practice to provide the safest, most up-to-date care. Student registered nurse anesthetists can use apps not only to familiarize themselves with procedures, but also to review standards of care, common anesthesia plans for a specific procedure, and drug dosage guidelines. This new technology is not only valuable for those early in their 
practice; apps can also be used by seasoned CRNAs to review such items as specific anesthesia considerations in uncommon or new procedures, anticoagulation guidelines with regards to procedures and regional anesthesia, and to learn about new drugs and treatment guidelines.

The use of apps should to be applied to the education of new SRNAs. Usage of specific apps could be initiated during the beginning of anesthesia school in order to ensure that all new practitioners are using a common resource to access the most up-todate information. It would also ensure that the SRNAs are using this technology appropriately. There may also need to be continuing education on new apps that are available and how they can be introduced into seasoned providers’ practice By joining committees in their hospitals, CRNAs can make their opinions known and educate colleges and administration on the importance of apps in clinical practice.

Policies should be in place to allow CRNAs and SRNAs to access smartphone apps to view important case specific information. With many institutions initiating no cellphone policies in patient care areas, these policies could negatively affect patient outcomes. However, special attention should be placed when writing policies to ensure that patient privacy and professionalism is maintained while allowing access to smartphones. Also, by being a member of the AANA, SRNAs and CRNAs can help to shape the way polices about anesthesia apps are written nationally.

Additional research on the role of smartphone apps in anesthesia is needed to understand how evolving technology affects patient care. Other key areas that should be researched are: CRNAs using anesthesia apps, smartphones as a distraction in the 
operating room, validity of anesthesia specific apps, and anesthesia apps being used by educators to teach SRNAs. Further research should be done to evaluate how often other practitioners are using apps in clinical practice. Also, the types of apps, and if there is a common app that is being used routinely in practice amongst different disciplines. It is important for APNs to incorporate new technologies into their practice but more research is needed to evaluate how to incorporate these technologies and to what extent they should be included into patient care. 


\section{References}

Dasari, K. B., White, S. M., \& Pateman, J. (2011). Survey of iPhone usage among anaesthetists in England. Anaesthesia; Journal of the Association of Anaesthetists of Great Britain and Ireland, 66(7), 630-631. http://doi:101111/j.13652044.2011.06747.x

Franko, O. I., \& Tirrell, T. F. (2012). Smartphone app use among medical providers in ACGME training programs. Journal of Medical Systems, 36(5), 3135-3139. https://doi10.1007/s10916-011-97987-7

Garrett, B., \& Klein, G. (2008). Value of wireless personal digital assistants for practice: Perceptions of advanced practice nurses. Journal of Clinical Nursing, 17(16), 2146-2154.

Gupta, R. K., \& McEvoy MD. (2017). Initial experience of the American Society of Regional Anesthesia and Pain Medicine coags Reginal Smartphone Application: A novel report of global distribution and clinical usage of an electronic decision support tool to enhance guideline use. Regional Anesthesia and Pain Medicine, 41(3), 334-338. https://doi.org/10.1097/AAP.0000000000000391

Hyman, I. E., Boss, M. S., Wise, M. B., Mckenzie, K. E., \& Caggiano, J. M. (2009). Did you see the unicycling clown: Inattentional blindness while walking and talking on a cellphone. Applied Cognitive Psychology, 24(5), https://doi:

10.1002/acp.1638 
Koivunen, M., Niemi, A., \& Hupli, M. (2015). The use of electronic devices for communication with colleagues and other healthcare professionals: nursing professionals’ perspectives. Journal of Advanced Nursing, 71(3), 620-631. https://doi:10.1111/jan.12529

Liguori, S., Stacchini, M., Ciofi, D., Olivini, N., Bisogni, S., \& Festini, F. (2016). Effectiveness of an app for reducing preoperative anxiety in children: A randomized clinical trial. JAMA Pediatrics, 170(8). http://doi:10.1001/jamapediatrics.2016.0533

Low, D., Clark, N., Soar, J., Padkin, A., Stoneham, A., Perkins, G. D., \& Nolan, J. (2011). A randomized control trial to determine if use of the iResus application on a smart phone improves the performance of an advanced life support provider in a simulated medical emergency. Journal of the Association of Anaesthesia of Great Britain and Ireland, 66(4), 255-262. https://doi:10.1111/j.13652044.2011.06649.x

McNally, G., Frey, R., \& Crossan, M. (2016). Nurse manager and student nurse perceptions of the use of personal smartphones or tablets and the adjunct applications, as an educational tool in clinical settings. Nurse Education in Practice, 23(2017), 1-7. http://dx.doi.org/10.1016/j.nepr.2016.12.004

National Safety Council. (2015). Annual estimate of cell phone crashes 2013. Retrieved from http://www.nsc.org/DistractedDrivingDocuments/Attributable-RiskEstimate.pdf 
Pınar, H. U., Karaca, O., Doğan, R., \& Konuk, Ü. M. (2015). Smartphone use habits of anesthesia providers during anesthetized patient care: a survey from Turkey. BMC Anesthesiology, 16(1), 88. http://doi.org/10.1186/s12871-016-0245-7

Putzer, G. J. \& Park, Y. (2010). The effects of innovation factors on smartphone adoption by nurses in community hospitals. Perspectives in Health Information Management, 7(winter), $1 \mathrm{~b}$.

Rogers, E. M. (2003). Diffusion of innovations. New York: Free Press.

Smith, T., Darling, E., \& Searles, B. (2011). 2010 Survey on cell phone use while performing cardiopulmonary bypass. Perfusion, 26(5), 375-380. https://doi:10.1177/0267659111409969

Steury, E. E. (2016). Mobile phone short message service to improve malaria pharmacoadherence in Zambia. Journal of Nursing Scholarship, 48(4), 354-361. 


\section{Appendix A}

\section{SMARTPHONE APPS IN ANESTHESIA: THE NEXT GENERATION Survey}

1. How many times per week do you use apps on your smart phone to assist in clinical practice?
A. 0
B. $1-3$
C. $4-6$
D. $7-10$
E. 10 or more

2. How many times in one week do you use apps for drug references?
A. 0
B. $1-3$
C. $4-6$
D. $7-10$
E. 10 or more

3. How many times per week do you use apps for case information (specific case tips or pointers)?
A. 0
B. $1-3$
C. $4-6$
D. $7-10$
E. 10 or more

4. How many times a week do you use pediatric specific anesthesia apps? If you do not do pediatric anesthesia, please put N/A.
A. 0 
B. $1-3$

C. $4-6$

D. $7-10$

E. 10 or more

F. N/A

5. How many times a week do you use apps for regional anesthesia? If you do not perform regional anesthesia, please indicate N/A
A. 0
B. $1-3$
C. $4-6$
D. $7-10$
E. 10 or more
F. N/A

6. Have you ever paid for an anesthesia app?
A. Yes
B. No

7. How many apps specific to your anesthesia practice do you have on your smartphone?
A. 0
B. $1-3$
C. $4-6$
D. $7-10$
E. 10 or more

8. Please list your most commonly used anesthesia apps. 